\title{
Sleep Duration and Snoring at Midlife in Relation to Healthy Aging in Women 70 Years of Age or Older
}

\author{
Hongying Shi $\mathbb{D}^{1,2}$ \\ Tianyi Huang ${ }^{3,4}$ \\ Yanan $\mathrm{Ma}^{5}$ \\ A Heather Eliassen ${ }^{2,3}$ \\ Qi Sun ${ }^{2-4}$ \\ Molin Wang $2,3,6$ \\ 'Department of Epidemiology and Health \\ Statistics, School of Public Health and \\ Management, Wenzhou Medical University, \\ Wenzhou, Zhejiang, People's Republic of \\ China; ${ }^{2}$ Department of Epidemiology, \\ Harvard T.H. Chan School of Public Health, \\ Boston, MA, USA; ${ }^{3}$ Channing Division of \\ Network Medicine, Department of \\ Medicine, Brigham and Women's Hospital, \\ Boston, MA, USA; ${ }^{4}$ Department of \\ Nutrition, Harvard T.H. Chan School of \\ Public Health, Boston, MA, USA; \\ ${ }^{5}$ Department of Biostatistics and \\ Epidemiology, School of Public Health, China \\ Medical University, Shenyang, Liaoning, \\ People's Republic of China; ${ }^{6}$ Department of \\ Biostatistics, Harvard T.H. Chan School of \\ Public Health, Boston, MA, USA
}

This article was published in the following Dove Press journal: Nature and Science of Sleep

Purpose: Both short and long sleep durations are associated with higher mortality. This study examined the association between sleep duration and overall health among those who survive to older ages.

Participants and Methods: In the Nurses' Health Study, participants without major chronic diseases in 1986 and survived to age 70 years or older in 1995-2001 were included. Habitual sleep duration and snoring were self-reported in 1986. Healthy aging was defined as being free of 11 major chronic diseases and having no cognitive impairment, physical impairment, or mental health limitations. Logistic regression was used to estimate the odds ratios (ORs) and 95\% confidence intervals (CIs) for healthy aging.

Results: Of the 12,304 participants, 1354 (11.0\%) achieved healthy aging. We observed a non-linear association between sleep duration and the odds of achieving healthy aging. Compared with women sleeping 7 hours per day, women with longer sleep duration were less likely to achieve healthy aging; there was also a suggestion of lower odds of healthy aging for shorter sleepers, although the associations did not reach statistical significance: the multivariate-adjusted ORs ( $95 \%$ CIs) of healthy aging for those sleeping $\leq 5,6,8$, and $\geq 9$ hours were $0.94(0.70,1.27), 0.88(0.76,1.02), 0.83(0.72,0.96)$, and $0.60(0.43,0.84)$, respectively. Similar non-linear associations were consistently observed for individual dimensions of healthy aging. Regular snoring was associated with $31 \%$ lower odds of healthy aging $(95 \% \mathrm{CI}: 0.54,0.88)$, which was primarily due to lower odds of having no major chronic diseases.

Conclusion: Both short and long sleep durations as well as regular snoring at midlife were associated with lower odds of healthy aging in later life.

Keywords: sleep, healthy aging, prospective studies, Nurses' Health Study, snoring

\section{Introduction}

Correspondence: Molin Wang Department of Epidemiology, Harvard T.H. Chan School of Public Health, 665

Huntington Avenue, Boston, MA, 02II5, USA

Tel + I6I75252202

Email mwang@hsph.harvard.edu

Qi Sun

Department of Nutrition, Harvard T.H.

Chan School of Public Health, 665

Huntington Avenue, Boston, MA, 02115 ,

USA

Tel + I 6174327490

Email qisun@hsph.harvard.edu
Life expectancy has increased over the last century, and 617 million people (8.5\%) are 65 years or older worldwide. ${ }^{1}$ By 2050 , this number is projected to reach 1.6 billion (nearly $20 \%$ ) of the world's population. ${ }^{2}$ However, longevity does not entail good health at older ages. A major challenge that aging societies are facing today is severe financial and societal burdens caused by diseases and disabilities prevailing in older populations. ${ }^{3}$ To address this challenge, the World Health Organization has identified financial, social, and scientific preparation for populalaunching a Healthy Longevity Global Grand Challenge, an international effort tion aging as a global imperative, and the US National Academy of Medicine is 
directed at improving the health for older people ${ }^{2}$ that emphasizes the urgent need of multidisciplinary solutions to maximize the number of years lived in good health. All these actions highlight the importance of achieving healthy aging, including being free of diseases and having good physical, mental and cognitive health. So far, prior studies have linked several lifestyle factors, ${ }^{4}$ such as physical activity, ${ }^{5,6}$ alcohol drinking, ${ }^{7}$ weight change, ${ }^{8}$ and diet quality, ${ }^{9}$ to healthy aging. However, more research is needed to identify other modifiable factors that may lead to healthy aging.

Sleep is a critical health behavior and plays a central role in human health. Emerging evidence shows that sleep duration may be an important risk factor for multiple chronic diseases, ${ }^{10-12}$ cognitive function, ${ }^{13,14}$ mental health, ${ }^{15}$ and mortality. ${ }^{16-19}$ Evidence synthesis based on meta-analysis generally supports that both short and long sleep durations are adversely associated with a wide range of health outcomes. Further, it has been increasingly recognized that sleep health is multi-dimensional beyond sleep duration. ${ }^{20}$ Snoring, which causes sleep disturbances and is a hallmark symptom of obstructive sleep apnea, has been associated with an increased risk of physical, cognitive and mental problems in adult populations..$^{21-23}$ Thus far, to the best of our knowledge, no prospective study has considered sleep duration or snoring as potentially modifiable risk factors for healthy aging. Moreover, sleep duration and snoring are closely related to other lifestyles such as physical activity, sedentary time, and overweight/ obesity, ${ }^{24,25}$ although few studies have explored their interrelationship or their joint associations with overall health.

In this study, we aimed to examine the independent associations of sleep duration and snoring with healthy aging as measured by a full spectrum of health outcomes, and explore whether and how other lifestyle factors modified these associations in the Nurses' Health Study.

\section{Participants and Methods Study Population}

The Nurses' Health Study (NHS) is a prospective cohort study of 121,701 US registered nurses aged 30-55 that was established in 1976 when a baseline questionnaire was administered to collect information of demographics, lifestyle and medical history. Follow-up questionnaires have been used to update these information and newly diagnosed diseases every 2 years since the study baseline. Women were first asked to report their sleep duration in
1986, which was treated as a baseline for the current analysis. The end of follow-up was through the year 2000 when the overall health status among survivors aged 70 years or older was evaluated, including chronic diseases, physical function, mental health, and cognitive function. The cumulative follow-up rate was over $95 \%$ in this cohort. ${ }^{6}$

The study was conducted in accordance with the Declaration of Helsinki. The study protocol was approved by the institutional review boards of the Brigham and Women's Hospital and the Harvard T.H. Chan School of Public Health. Participants provided implied consent by returning the questionnaires and we also obtained written consent from each participant to obtain and review medical records.

\section{Assessment of Sleep Habits}

Women reported their sleep habits in 1986, including daily sleep duration and frequency of snoring. Participants were asked to specify the average total hours of sleep in 24 hours; possible choices included $\leq 5,6,7,8,9,10$, and $\geq 11$ hours. Considering the small sample size of the last two groups, we collapsed the last three categories, leaving five categories of sleep duration for data analyses: $\leq 5,6,7,8$, and $\geq 9$ hours. We defined regular snorers as those who reported snoring regularly and non-snorers as those who only occasionally or almost never snored.

The self-reported sleep duration strongly correlated with sleep duration recorded in sleep diaries $\left(\mathrm{r}_{\mathrm{s}}=0.79\right.$, $P<0.001$ ), and details of the validity and reproducibility of self-reported sleep have been reported elsewhere. ${ }^{26}$

\section{Assessment of Healthy Aging}

The definition of healthy aging has been described in previous publications. ${ }^{6,7}$ In brief, the outcome is defined based on survival status until at least age 70 years and health information from four domains including chronic diseases, cognitive function, physical function, and mental health. A description of each of the four domains is listed below.

\section{Assessment of Cognitive Function}

Beginning in 1995 through 2001, every participant who had survived to age 70 was invited to a cognitive function study and 19,415 (92\% of 21,202 eligible nurses) women agreed to participate. We administered the Telephone Interview of Cognitive Status (TICS), a telephone version of the Mini-Mental State Examination (MMSE). TICS 
scores range from 0 (worst) to 41 (best), and a score below 31 is considered as cognitive impairment. ${ }^{27}$ Studies have shown high test-retest reliability and validity of TICS in assessing cognitive status. For example, a strong correlation $(r=0.97)$ was found between TICS and MMSE assessments. ${ }^{28}$ Trained study nurses who were unaware of the study hypothesis performed the assessment, and inter-interviewer reliability was high $(>0.95){ }^{29}$

\section{Assessment of Chronic Diseases}

Clinical diagnoses of major chronic diseases were queried on each biennial questionnaire since 1986, including cancer, diabetes, myocardial infarction, coronary artery bypass graft surgery or percutaneous transluminal coronary angioplasty, congestive heart failure, stroke, kidney failure, chronic obstructive pulmonary disease, Parkinson disease, multiple sclerosis, and amyotrophic lateral sclerosis. Self-reported disease diagnosis was confirmed by study physicians through medical record review, pathology report review, telephone interview, or supplementary questionnaire inquiries, and has been shown to have high validity in this cohort. ${ }^{6}$ Women who reported no history of these 11 diseases as of 2000 were considered being free of chronic diseases.

\section{Assessment of Physical Function and Mental Status}

Physical function and mental health were assessed by the Medical Outcomes Study Short-Form Health Survey (SF36), which was embedded in the 1992, 1996, and 2000 follow-up questionnaires. Physical function was evaluated by 10 questions on physical limitations in performing the following activities: moderate activities; bathing and dressing yourself; walking 1 block; walking several blocks; walking more than 1 mile; vigorous activities; bending, kneeling, or stooping; climbing 1 flight of stairs; climbing several flights of stairs; and lifting or carrying groceries. Each question had 3 response choices: "Yes, limited a lot," "Yes, limited a little," or "No, not limited at all." Impairment of physical function was defined as limited at least "a little" on moderate activities or limited "a lot" on more difficult physical tasks; otherwise, they were defined as no impairment of physical function.

Mental health was evaluated by five questions: "have you been a very nervous person?, have you felt so down in the dumps nothing could cheer you up?, have you felt calm and peaceful?, have you felt downhearted and blue?, and have you been a happy person?" There were six possible responses to each question, ranging from "none of the time" to "all the time." Based on the response to these questions, a score between 1 and 6 was assigned to each question, with the score 6 indicating the best mental status and score 1 indicating the worst. We then summed these scores and rescaled them to a range of 0-100. Good mental health was defined as a mental health score greater than 84 (the median value in this cohort). ${ }^{6}$ The validity and reproducibility of the SF-36 and its components have been previously established. ${ }^{30}$

\section{Covariates}

The baseline and subsequent questionnaires collect information of a broad range of covariates, including demographic characteristics such as age, education, husband's education, race, marital status; lifestyle factors, such as body mass index (BMI, $\mathrm{kg} / \mathrm{m}^{2}$ ), smoking status, physical activity, sitting time, and standing time; family history of cancer, family history of myocardial infarction, and family history of diabetes; hypertension and high cholesterol; use of supplemental multivitamin, menopausal status and postmenopausal hormone use, and aspirin use. Median annual household income and home value were estimated from the census tract of participant's residence, geocoded to the 1994 US Census. Dietary data were collected using a validated semi-quantitative food frequency questionnaire, ${ }^{31,32}$ based on which, total energy intake (kJ/day), alcohol (g/day) and coffee (cups/day) consumption and the Alternate Healthy Eating Index (AHEI) were derived. ${ }^{33}$ In addition, women were asked the total number of years working rotating night shifts (at least 3 nights per month) with 8 pre-specified categories, including never, $1-2,3-5,6-9,10-14,15-19,20-29$, and $\geq 30$ years. Participants were also asked for "What is your usual sleeping position?" with three response categories: on back, on side, or on front.

\section{Study Population for Analysis}

Of the 19,415 participants who completed the assessment of cognitive function, we excluded participants who had missing year of birth $(n=20)$, who reported any of the 11 chronic diseases $(n=3893)$ at or before baseline. We further excluded those who skipped more than 2 items on mental health or 5 items on physical function scale in SF-36 $(n=708)$, those with no questionnaire in $1986(n=773)$, and those with missing sleep information in 1986 $(n=1737)$, a total of 12,304 participants remained for this 
analysis. Participants who did not answer the sleep question did not differ substantially from respondents according to age, BMI, physical activity, alcohol consumption, and other major potential confounders (Table S1). In a secondary analysis, we further included 2425 women who died before 2000 as usual agers $(n=14,729)$.

\section{Statistical Methods}

First, we used logistic regression models to estimate odds ratios (OR) and 95\% confidence intervals (95\% CI) by sleep duration $[\leq 5,6,7$ (reference), 8 , and $\geq 9$ hours per 24 hours]. An OR $<1$ in these models indicates decreased odds of healthy aging. In the multivariable analysis, we adjusted for possible confounding factors at baseline, including age (continuous), education, race, marital status, household income, family history of diseases (cancer, myocardial infarction, and diabetes), hypertension and high cholesterol, use of supplemental multivitamin (yes, no), menopausal status and hormone use (premenopausal, postmenopausal never users, postmenopausal past users, postmenopausal current users), aspirin use (regular use or not), smoking history (never, former smoker, current smoker), alcohol intake (none, 1-14.9, $\geq 15 \mathrm{~g} / \mathrm{d}$ ), total energy intake, diet quality, coffee consumption, physical activity (MET-hours/week), standing and sitting time (all in quintiles). Because body mass index (BMI) had a strong and positive association with risk of healthy aging, ${ }^{34}$ we further adjusted for BMI $(<18.5,18.5-24.9,25-29.9$, $\geq 30 \mathrm{~kg} / \mathrm{m}^{2}$ ) in a separate model. We used restricted cubic splines with 4 knots to evaluate the possible nonlinearity of the association between sleep duration in midlife (measured in 1986) and healthy aging. ${ }^{35}$ We tested nonlinearity in the dose-response relationship by comparing the model with only the linear term to the model with the linear and the cubic spline terms using the likelihood ratio test. Likewise, we examined the dose-response curves for the relationship between sleep habits and four individual components of healthy aging.

Stratified analyses were performed to evaluate whether the association with sleep duration was modified by baseline lifestyle variables, including $\operatorname{BMI}\left(<25, \geq 25 \mathrm{~kg} / \mathrm{m}^{2}\right)$, smoking behavior (never, smokers), alcohol consumption (none, 1-14.9, $\geq 15 \mathrm{~g} / \mathrm{d}$ ), physical activity (<median, $\geq$ median), diet quality ( $<$ median, $\geq$ median), total calorie intake $(<$ median, $\geq$ median), standing and sitting time ( $<$ median, $\geq$ median). Statistical interactions were assessed by likelihood ratio tests comparing the models with and without the multiplicative interaction terms. In addition, we examined the joint associations of sleep duration and regular snoring.

We performed several sensitivity analyses to examine the robustness of observed associations. First, we repeated all analyses including 2425 women who died before age 70 through 2000 as usual agers. Then, we restricted analyses within participants with sleep position on side and also those with no history of snoring. Because shift work status might be a substantial confounder due to its potentially strong associations with both sleep duration and overall health, we excluded women who reported having rotating shift work in 1992 (at least 3 nights/month in addition to days or evenings during the last six months), and also restricted analysis within women who reported never having shift work in 1988. And additional adjustment for shift work also was performed.

All analyses were performed with SAS, version 9.4 (SAS Institute, Inc., Cary, North Carolina). A 2-sided $P$ value of less than 0.05 was considered statistically significant.

\section{Results}

Of the 12,304 participants included in the analysis, 8321 $(67.6 \%)$ women remained free of the 11 chronic diseases included in the definition of healthy aging, 3434 (27.9\%) had no impairment of physical function, 5311 (43.2\%) had good mental health, and $11,079(90.0 \%)$ reported no impairments of cognitive function during the follow-up assessment in 1995-2001. A total of 1354 (11.0\%) participants met all criteria of healthy aging and therefore were healthy survivors, and the rest were usual agers. Compared with usual agers (Table 1), healthy survivors were more active, had lower BMI, and were less likely to be smokers or have hypertension and high cholesterol. Healthy agers were also less likely to be regular snorer compared with usual agers (6.0\% vs $10.8 \%)$, but they had a similar mean sleep duration at baseline $(7.0 \pm 1.0 \mathrm{~h}$ vs $6.9 \pm 0.9 \mathrm{~h})$.

\section{Sleep Duration and Healthy Aging}

Table 2 summarizes the age- and multivariate-adjusted ORs of healthy aging associated with sleep duration. After adjustment for all covariates except for BMI, compared with women sleeping 7 hours per day, women with longer sleep duration were less likely to achieve healthy aging; there was also a suggestion of lower odds of healthy aging for shorter sleepers, although the associations did not reach statistical significance: ORs (95\% CIs) for those sleeping $\leq 5,6,8$, and $\geq 9$ hours were $0.93(0.69,1.24), 0.87(0.75,1.01), 0.83$ (0.71, 
Table I Baseline Characteristics of Healthy Agers and Usual Agers in the Nurses' Health Study

\begin{tabular}{|c|c|c|}
\hline Characteristics & Usual Agers $(n=10,950) *$ & Healthy Agers $(n=\mid 354) *$ \\
\hline Age at 1986, years $^{\dagger}$ & $60.6(2.5)$ & $60.1(2.5)$ \\
\hline \multicolumn{3}{|l|}{ Education, \% } \\
\hline Registered nurse & 77.9 & 73.4 \\
\hline Bachelor & 15.8 & 17.5 \\
\hline Master or doctorate & 6.4 & 9.1 \\
\hline \multicolumn{3}{|l|}{ Husband's education, \% } \\
\hline$\leq$ High school & 52.2 & 46.1 \\
\hline College graduate & 27.1 & 30.2 \\
\hline Graduate school & 20.6 & 23.7 \\
\hline Race (white), \% & 98.5 & 98.3 \\
\hline \multicolumn{3}{|l|}{ Marital status, \% } \\
\hline Married & 92.8 & 92.9 \\
\hline Widowed & 4.6 & 4.9 \\
\hline Separated, divorced, or never married & 2.6 & 2.2 \\
\hline Family annual income, $10,000 \$$ & $5.7(4.5,7.3)$ & $6.0(4.7,7.7)$ \\
\hline Family home value, $10,000 \$$ & $12.8(8.9,19.5)$ & $13.6(9.3,21.2)$ \\
\hline $\mathrm{BMI}, \mathrm{kg} / \mathrm{m}^{2}$ & $25.5(4.4)$ & $23.8(3.2)$ \\
\hline \multicolumn{3}{|l|}{ Smoking status, \% } \\
\hline Never smoker & 47.2 & 53.5 \\
\hline Past smoker & 35.8 & 35.3 \\
\hline Current smoker & 16.9 & 11.2 \\
\hline \multicolumn{3}{|l|}{ Alcohol intake $(g / d), \%$} \\
\hline None & 42.1 & 38.6 \\
\hline $1-14.9$ & 45.4 & 48.3 \\
\hline$\geq 15$ & 12.6 & 13.1 \\
\hline AHEI (no alcohol) & $48.9(10.7)$ & $51.3(11.1)$ \\
\hline Total calories, kcal & I $757.4(520.2)$ & $1728.4(527.3)$ \\
\hline Total coffee, cup/d & $2.5(1.0,2.9)$ & $2.5(1.0,3.5)$ \\
\hline Physical activity, MET-h/wk ${ }^{\ddagger}$ & $7.9(2.9,19.4)$ & II.6 (4.3, 26.2) \\
\hline Standing time, $\mathrm{h} / \mathrm{d}$ & $4.4(I .1,7.2)$ & $4.4(1.6,7.2)$ \\
\hline Sitting time, $\mathrm{h} / \mathrm{d}$ & $4.4(I .1,4.4)$ & $2.2(I .1,4.4)$ \\
\hline Family history of diabetes, $\%$ & 29.6 & 26.4 \\
\hline Family history of myocardial infarction, \% & 17.2 & 15.5 \\
\hline Family history of cancer, $\%$ & 18.3 & 16.0 \\
\hline History of hypertension, \% & 21.0 & 13.3 \\
\hline History of high cholesterol, \% & 10.4 & 7.5 \\
\hline Use of multivitamin, \% & 43.8 & 41.3 \\
\hline \multicolumn{3}{|l|}{ Menopausal status and hormone use, \% } \\
\hline Pre-menopausal & 1.3 & 1.0 \\
\hline Post and never used & 73.2 & 73.8 \\
\hline Post and past user & 3.6 & 4.0 \\
\hline Post and current user & 22.0 & 21.2 \\
\hline Regular aspirin use ( $\geq 2$ tabs/wk), $\%$ & 38.0 & 26.6 \\
\hline \multicolumn{3}{|l|}{ Sleep habits } \\
\hline Sleep duration, h/d & $7.0(1.0)$ & $6.9(0.9)$ \\
\hline Regular snorer, $\%$ & 10.8 & 6.0 \\
\hline Night shift work $>5$ years, $\%$ & 21.4 & 16.0 \\
\hline \multicolumn{3}{|l|}{ Sleep position, \% } \\
\hline On back & 6.2 & 6.6 \\
\hline On side & 84.4 & 82.6 \\
\hline On front & 9.4 & 10.8 \\
\hline
\end{tabular}

Notes: *Values are mean $(S D)$ or median $\left(P_{25}, P_{75}\right)$ for continuous variables and percentages for categorical variables and are standardized to the age distribution of the study population. ${ }^{\dagger}$ Value was not age adjusted. ${ }^{\ddagger} \mathrm{MET}-\mathrm{h} / \mathrm{wk}=$ sum of the average time/week spent in each activity $\times$ MET value of each activity.

Abbreviations: BMI, body mass index (calculated as weight in kilograms divided by the square of height in meters); MET, metabolic-equivalent; AHEl, Alternate-Healthy Eating Index. 
Table 2 Odds Ratios of Healthy Aging by Sleep Duration, Snoring at Midlife in the Nurses' Health Study*

\begin{tabular}{|c|c|c|c|c|c|}
\hline & $\begin{array}{l}\text { Number of } \\
\text { Participants }\end{array}$ & $\begin{array}{r}\text { Healthy Aging, } \\
\text { n (\%) }\end{array}$ & Age-Adjusted & MVI-Adjusted $^{\dagger}$ & MV2-Adjusted ${ }^{\ddagger}$ \\
\hline \multicolumn{6}{|l|}{ Sleep duration, hours } \\
\hline$\leq 5$ & 506 & $57(11.3)$ & $0.91(0.68,1.21)$ & $0.93(0.69,1.24)$ & $0.94(0.70,1.27)$ \\
\hline 6 & 3052 & $326(10.7)$ & $0.85(0.74,0.98)$ & $0.87(0.75,1.01)$ & $0.88(0.76,1.02)$ \\
\hline 7 & 5061 & $621(12.3)$ & I (reference) & I (reference) & I (reference) \\
\hline 8 & 3094 & $310(10.0)$ & $0.8 \mathrm{I}(0.70,0.93)$ & $0.83(0.7 \mathrm{I}, 0.96)$ & $0.83(0.72,0.96)$ \\
\hline$\geq 9$ & 591 & $40(6.8)$ & $0.54(0.38,0.75)$ & $0.59(0.42,0.82)$ & $0.60(0.43,0.84)$ \\
\hline \multicolumn{6}{|l|}{ Regular snoring } \\
\hline No & 10,974 & $1259(\mid 1.5)$ & I (reference) & I (reference) & I (reference) \\
\hline Yes & 1254 & $81(6.5)$ & $0.53(0.42,0.67)$ & $0.6 \mathrm{I}(0.49,0.78)$ & $0.69(0.54,0.88)$ \\
\hline
\end{tabular}

Notes: *ORs <1.00 denote lower odds of healthy aging. For sleep duration, $n=13,099$; for snoring: $n=\mid 3,018$ due to 81 participants with missing snoring; we defined regular snorers as those who reported snoring regularly and non-snorers as those who only occasionally or almost never snored; ${ }^{\dagger} \mathrm{MVI}$ was adjusted for age (continuous), education (registered nurse, bachelor, or graduate), marital status (married, widowed, or separated/divorced), household income (quintiles); family history of cancer, family history of myocardial infarction and family history of diabetes (yes or no); baseline hypertension and high cholesterol (yes or no); menopausal status and hormone use (premenopausal, postmenopausal never users, postmenopausal past users, postmenopausal current users), aspirin use (regular use or not); smoking history (never, former smoker, current smoker), alcohol intake (none, I-I4.9, $\geq 15 \mathrm{~g} / \mathrm{d}$ ), total energy intake (quintiles), diet quality (Alternate Healthy Eating Index score, in quintiles), physical activity (metabolic equivalent task-hours per week, in quintiles), standing and sitting time (in quintiles); ${ }^{\ddagger} \mathrm{MV} 2$ : MVI+ body mass index (BMI <I8.5, $18.5-24.9,25-29.9, \geq 30 \mathrm{~kg} / \mathrm{m}^{2}$ ).

0.96), and $0.59(0.42,0.82)$, respectively. The associations remained similar with additional adjustment for BMI. As shown in the estimated dose-response curve from a multivariate analysis (Figure 1A), there was a nonlinear association between sleep duration and healthy aging ( $P$ for nonlinearity $=0.0073$ ), with substantially lower odds of healthy aging for long sleep duration.

When examining the association by each domain of healthy aging (Table 3), short sleep duration was associated with lower odds of no limitation of mental health (OR for $\leq 5$ versus 7 hours: 0.73 ; 95\% CI: $0.60,0.88$ ) and no impairment of cognitive function (comparable OR: 0.67; 95\% CI: 0.51, 0.88), whereas long sleep duration was associated with lower odds of good physical function (OR for $\geq 9$ versus 7 hours: 0.69 ; 95\% CI: $0.56,0.86$ ), no limitation of mental health (comparable OR: 0.83 ; 95\% CI: $0.69,0.99)$ and no impairment of cognitive function (comparable OR: 0.74; 95\% CI: 0.57 , $0.97)$. The results were further supported by the significant nonlinear associations of sleep duration with no impairment of physical function $(P$ for nonlinearity $=0.0004$ ), no mental health limitations ( $P$ for nonlinearity $=0.0002$ ), and no impairment of cognitive function ( $P$ for nonlinearity $=0.0336)$ in the restricted cubic spline analyses (Figure $1 \mathrm{C}-\mathrm{E}$ ). However, no significant association was observed between sleep duration and the odds of having no major chronic disease ( $P$ for linearity $=0.1064$; Figure 1B).

\section{Snoring and Healthy Aging}

We found that regular snoring was associated with reduced odds of healthy aging after adjusting for potential covariates except BMI (OR: 0.61; 95\% CI: 0.49, 0.78; Table 2). Further adjustment of BMI somewhat attenuated the association (OR: $0.69,95 \% \mathrm{CI}: 0.54,0.88$ ). The associations of snoring with each domain of healthy aging are shown in Table 3. Regular snoring was associated with 14\% lower odds of being free of major chronic diseases (95\% CI: $0.76,0.97)$, but was not significantly associated with the other three domains of healthy aging.

When we examined sleep duration and snoring jointly, participants with long sleep ( $\geq 9 \mathrm{~h} /$ day) and regular snoring had the lowest odds of healthy aging compared with participants with 7 hours of sleep and no regular snoring (fully adjusted OR: 0.23; 95\% CI: 0.06, 0.94; Figure 2). However, we did not observe a significant interaction between sleep duration and snoring on healthy aging ( $P$ for interaction $=0.198)$.

\section{Stratified Analysis by Lifestyles}

In stratified analyses of sleep duration and healthy aging by lifestyle factors, including overweight, smoking status, alcohol intake, total energy intake, dietary quality, physical activity, standing and sitting time (Table S2), long sleep duration was consistently associated with lower odds of healthy aging almost in each subgroup (although some of these associations were not statistically significant due to 

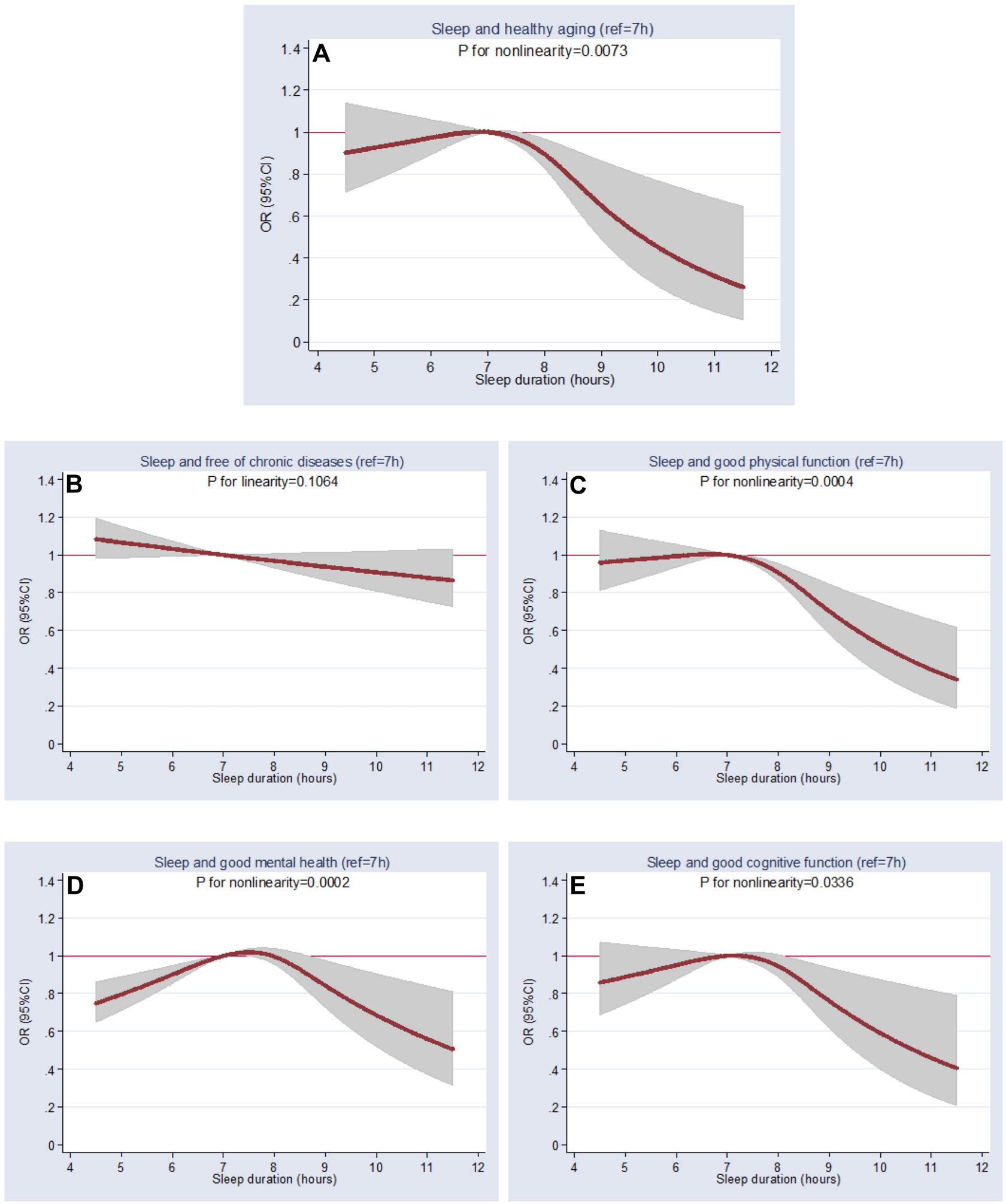

Figure I Association of sleep duration with healthy aging including 4 domains*. (A) Sleep duration and healthy aging, (B) sleep duration and free of chronic diseases, (C) sleep duration and good physical function, (D) sleep duration and good mental health, (E) sleep duration and good cognitive function. Notes: *ORs $<1.00$ denote lower odds of healthy aging. All model was adjusted for age (continuous), education (registered nurse, bachelor, or graduate), marital status (married, widowed, or separated/ divorced), household income (quintiles); family history of cancer, family history of myocardial infarction and family history of diabetes (yes or no); baseline hypertension and high cholesterol (yes or no); menopausal status and hormone use (premenopausal, postmenopausal never users, postmenopausal past users, postmenopausal current users), aspirin use (regular use or not); smoking history (never, former smoker, current smoker), alcohol intake (none, $1-14.9, \geq 15 \mathrm{~g} / \mathrm{d}$ ), total energy intake (quintiles), diet quality (Alternate Healthy Eating Index score, quintiles), physical activity (MET-h/wk), standing and sitting time (all in quintiles); body mass index (BMI <18.5, $18.5-24.9,25-29.9, \geq 30$ $\left.\mathrm{kg} / \mathrm{m}^{2}\right)$. 
Table 3 Odds Ratios of Each Domain of Healthy Aging by Sleep Duration, Snoring at Midlife in the Nurses' Health Study*

\begin{tabular}{|c|c|c|c|c|c|c|c|c|}
\hline & \multicolumn{2}{|c|}{ Free of Chronic Diseases } & \multicolumn{2}{|c|}{ Good Physical Function } & \multicolumn{2}{|c|}{$\begin{array}{c}\text { No Limitation of Mental } \\
\text { Health }\end{array}$} & \multicolumn{2}{|c|}{$\begin{array}{l}\text { No Impairment of } \\
\text { Cognitive Function }\end{array}$} \\
\hline & n (\%) & MV2-Adjusted & n (\%) & MV2-Adjusted & n (\%) & MV2-Adjusted & n (\%) & MV2-Adjusted \\
\hline \multicolumn{9}{|l|}{ Sleep duration, hours } \\
\hline$\leq 5$ & $342(67.6)$ & $1.01(0.83,1.23)$ & I4I (27.9) & $0.96(0.78,1.19)$ & $189(37.4)$ & $0.73(0.60,0.88)$ & $436(86.2)$ & $0.67(0.5 \mathrm{I}, 0.88)$ \\
\hline 6 & $2090(68.5)$ & $1.05(0.95,1.16)$ & $854(28.0)$ & $0.94(0.85,1.05)$ & $1268(41.6)$ & $0.87(0.79,0.95)$ & $2770(90.8)$ & $1.04(0.89,1.22)$ \\
\hline 7 & $3440(68.0)$ & I (reference) & $1516(30.0)$ & I (reference) & $2283(45.1)$ & I (reference) & $4592(90.7)$ & I (reference) \\
\hline 8 & $2075(67.1)$ & $0.98(0.89,1.08)$ & $802(25.9)$ & $0.86(0.77,0.96)$ & $|34|(43.3)$ & $0.95(0.86,1.04)$ & $2766(89.4)$ & $0.89(0.77,1.04)$ \\
\hline$\geq 9$ & $374(63.3)$ & $0.90(0.75,1.08)$ & $|2|(20.5)$ & $0.69(0.56,0.86)$ & $230(38.9)$ & $0.83(0.69,0.99)$ & $515(87.1)$ & $0.74(0.57,0.97)$ \\
\hline \multicolumn{9}{|l|}{ Regular snoring } \\
\hline No & $7502(68.4)$ & I (reference) & $3139(28.6)$ & I (reference) & $4764(43.4)$ & I (reference) & $9885(90.1)$ & I (reference) \\
\hline Yes & $766(6 I .1)$ & $0.86(0.76,0.97)$ & $270(21.5)$ & $0.92(0.79,1.07)$ & $509(40.6)$ & $0.95(0.84,1.08)$ & $1125(89.7)$ & $0.95(0.78,1.16)$ \\
\hline
\end{tabular}

Note: *All footnotes are same with Table 2.

small number of cases). The nonlinear association pattern between sleep duration and healthy aging appeared more apparent among those with longer sitting time $(P$ for interaction $=0.007)$ or standing time $(P$ for interaction $=0.044)$.

\section{Sensitivity Analysis}

We conducted further analyses to test the robustness of the association between sleep duration and healthy aging. In a secondary analysis, we included 2425 women who died before age 70 through 2000 as usual agers and repeated the analysis, and the results remained similar (Table S3). In

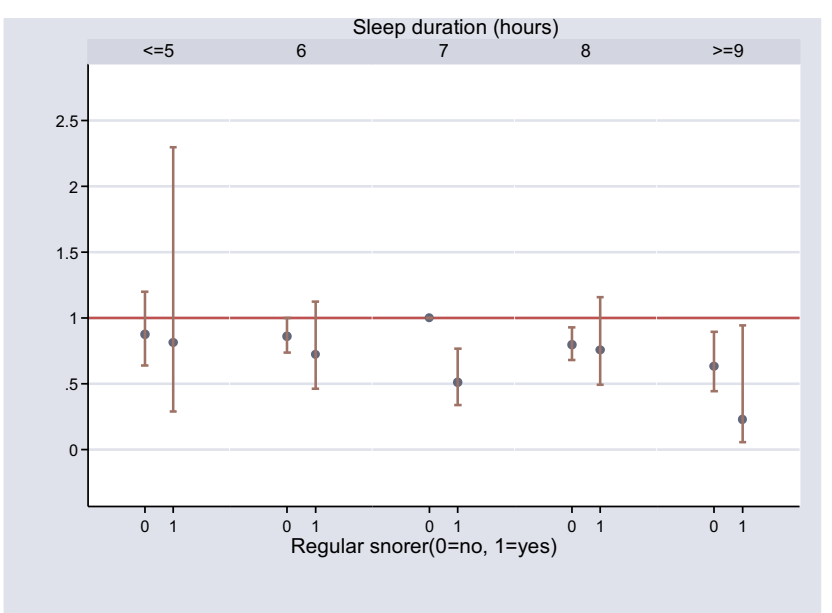

Figure 2 Joint effect of sleep duration and regular snoring on healthy aging*. Notes: *Model was adjusted for age (continuous), education (registered nurse, bachelor, or graduate), marital status (married, widowed, or separated/divorced), household income (quintiles); family history of cancer, family history of myocardial infarction and family history of diabetes (yes or no); baseline hypertension and high cholesterol (yes or no); menopausal status and hormone use (premenopausal, postmenopausal never users, postmenopausal past users, postmenopausal current users), aspirin use (regular use or not), smoking history (never, former smoker, current smoker), alcohol intake (none, I-14.9, $\geq 15 \mathrm{~g} / \mathrm{d}$ ), total energy intake (quintiles), diet quality (Alternate Healthy Eating Index score, quintiles), physical activity (MET-h/wk), standing and sitting time (all in quintiles); body mass index (BMI <18.5, $18.5-24.9,25-29.9, \geq 30 \mathrm{~kg} / \mathrm{m}^{2}$ ). a sensitivity analysis, excluding women with a history of shift work or recent shift work did not change our results materially (Table S4). Additional adjustment for duration of shift work also resulted in similar associations. Likewise, when we restricted analyses to the participants whose sleep position was on sides, and those who did not snore regularly, results were essentially unchanged (Table S4). The results did not appreciably change in sensitivity analyses with adjustment for continuous BMI instead of categorical BMI (data not shown). When sleep duration and snoring were mutually adjusted in the same model, the results for healthy aging and individual domains also remained the same (data not shown).

\section{Discussion}

In this large, prospective cohort study, sleep durations were non-linearly associated with odds of healthy aging after adjusting for potential confounding factors: in comparison with 7 hours of sleep, long sleep duration was associated with lower odds of achieving healthy aging at age 70 ; there was also a suggestion of lower odds of healthy aging for shorter sleepers, although the associations did not reach statistical significance. Such nonlinear relationships were also observed for different components of healthy aging, except for the absence of chronic diseases. Regular snoring was associated with reduced odds of healthy aging (especially free of diseases), independent of sleep duration. To our knowledge, this is the first study that characterized the associations of sleep habits with overall health status among those who have survived to older ages.

\section{Comparison with Others and Explanations}

The non-linear association observed in the current analysis was consistent with those observed in most prior studies in 
general documenting that both shorter and longer sleep durations were associated with increased mortality. ${ }^{16-18}$ For example, several meta-analyses showed a U-shaped association between sleep duration and mortality, with the lowest mortality observed among individuals with approximately 7-hour daily sleep duration. ${ }^{16-18}$ Studies also found U-shaped relationships between sleep duration and other health outcomes, including chronic diseases ${ }^{10-12}$ mental health ${ }^{15}$ memory or cognition decline, ${ }^{13,14}$ although the sleep duration was associated with the lowest risk varied from 6 to 9 hours for different disease outcomes. ${ }^{10,13,14,18,36}$ Interestingly, while short sleep duration has been previously associated with increased risk of diabetes, ${ }^{37}$ coronary heart disease ${ }^{38}$ and mortality ${ }^{26}$ in our cohort, we observed no significant association especially between short sleep duration and the composite chronic disease outcome, which may be driven by the null findings for cancers. ${ }^{39}$ In our study, we found self-reported sleep duration of 7 hours per night at midlife was associated with the highest odds of achieving healthy aging as well as optimal cognitive, physical, and mental health. This is consistent with recommended sleep duration of 7-8 hours per night by the American Academy of Sleep Medicine and highlights the potential benefits of adequate sleep duration on healthy aging.

There are several potential mechanisms for the nonlinear association. Given that sleep is essential for repair, restoration and rejuvenation of the human body, insufficient sleep may lead to alterations in these physiologic processes that ultimately precipitate the development of physical, mental and cognitive impairment. For example, sleep has been shown to enhance clearance of neurotoxic waste products in the brain, whereas sleep deprivation results in cerebral accumulation of $\beta$-amyloid, a key biomarker for cognitive decline and Alzheimer's disease. ${ }^{40-42}$ This may explain the associations with short sleep duration we observed for the domains of mental and cognitive health. Long sleep duration, on the other hand, may be the consequence of poor sleep quality due to frequent awakenings, which also disrupt these sleep-facilitated processes. Further, both short and long sleep durations are associated with energy imbalance, elevated inflammation, reduced immune response and insulin resistance; these pathways are key mechanisms for a variety of adverse health outcomes. Individuals with short or long sleep duration are also more likely to be affected by circadian disruption due to night shift work or irregular sleep schedules. Previous studies have linked shift work and irregular sleep schedules to increased risk of cardiometabolic disease and mental disorders. ${ }^{43-47}$ However, we cannot fully exclude the possibility that certain preclinical, unrecognized conditions for aging may result in extreme sleep duration.

Our results also suggest that, independent of sleep duration, snoring was associated with lower odds of healthy aging (especially free of chronic diseases). This was consistent with previous studies that have reported associations between snoring and chronic diseases such as hypertension, diabetes and cardiovascular events. ${ }^{48-50}$ Snoring is one of the cardinal manifestations of obstructive sleep apnea, ${ }^{51}$ which has been associated with chronic diseases, ${ }^{52-55}$ cancers, ${ }^{56}$ depression, ${ }^{57}$ and cognitive function. ${ }^{58}$ Therefore, habitual snorers are more likely to experience intermittent hypoxia, which results in reduced cerebral blood flow and brain oxygen supply during sleep and may adversely influence brain regions regulating mood and memory. ${ }^{59,60}$ Heavy snoring may also lead to repeated arousals and fragmented sleep that disrupt sleep quality and stimulate the sympathetic nervous system. ${ }^{61}$ Increased sympathetic activity is associated with the development of a wide range of health outcomes, ${ }^{62,63}$ including cardiometabolic disease, cancers and psychiatric disorders. Additionally, we found that participants with long sleep duration and regular snoring had the lowest odds of healthy aging. Although replication in future studies is warranted, our findings suggest potential target populations for sleep hygiene interventions to maximize the benefits of sleep on healthy aging.

\section{Limitation}

Several limitations of this study should be considered. First, self-reported sleep duration may be subjected to measurement error. However, previous validation studies found that self-reported usual sleep duration is well correlated with that derived from sleep diaries, ${ }^{26}$ and there was a moderate correlation between self-reported and actigraphy-assessed sleep duration. ${ }^{64,65}$ In addition, as our sleep data were prospectively collected, the measurement error was likely non-differential, biasing the association towards the null. Second, as this is an observational study, we cannot rule out the possibility of residual unmeasured confounding. For example, the observed associations may be confounded by sleep quality given its relationships with mortality ${ }^{66}$ and cognitive function. ${ }^{36}$ Third, we only assessed sleep habits once at baseline, which may not reliably reflect long-term sleep patterns. Future studies 
using repeated measures to evaluate changes or trajectory of sleep duration will provide further insights into the associations with healthy aging. ${ }^{67}$

Fourth, we could not entirely exclude the possibility of reverse causation as we did not assess impaired physical function, mental health, and subjective memory at study baseline. Thus, the study population may include individuals with these conditions that may lead to excess sleep duration or snoring. However, we excluded all people with major diseases at baseline and adjusted for common health conditions (eg, hypertension, high cholesterol) as covariates in the analysis, which often coexist with the aforementioned conditions. Finally, the participants in our study are predominantly white female nurses. Whether our results apply to men, other racial/ethnic groups, or people with heterogeneous socioeconomic backgrounds requires further study.

\section{Conclusion}

In conclusion, we found that sleep duration at midlife was nonlinearly associated with healthy aging in later life. In comparison with 7 hours of sleep, shorter and longer durations were associated with lower odds of achieving healthy aging at age 70. In addition, regular snorers had lower odds of attaining optimal health at old ages, especially for those with sleep duration $\geq 9$ hours. These findings highlight the role of sleep duration and snoring in promoting healthy longevity. Future research is necessary to better understand the mechanisms by which sleep duration and snoring, as well as other sleep traits, may influence healthy aging.

\section{Ethics Approval and Consent to Participate}

The study protocol was approved by the institutional review boards of the Brigham and Women's Hospital and the Harvard T.H. Chan School of Public Health. Participants provided implied consent by returning the questionnaires and we also obtained written consent from each participant to obtain and review medical records.

IRB protocol title and number: Improving Women's Health: The Nurses' Health Study, 1999-P-011114.

\section{Acknowledgments}

We would like to thank the participants and staff of the Nurses' Health Study for their valuable contributions.

\section{Author Contributions}

All authors made substantial contributions to conception and design, acquisition of data, or analysis and interpretation of data; took part in drafting the article or revising it critically for important intellectual content; agreed to submit to the current journal; gave final approval of the version to be published; and agree to be accountable for all aspects of the work. Hongying Shi and Tianyi Huang contributed equally as co-first authors and Qi Sun and Molin Wang authors contributed equally as co-corresponding authors.

\section{Funding}

The Nurses' Health Study was supported by the National Institutes of Health (grant number UM1CA186107). Dr. Shi is supported by Zhejiang Provincial Philosophy and Social Sciences Planning Project (21NDJC013Z). Dr. Huang is supported by the National Institutes of Health (K01HL143034). The funding sources did not participate in the design or conduct of the study; collection, management, analysis or interpretation of the data; or preparation, review, or approval of the manuscript.

\section{Disclosure}

Dr A Heather Eliassen reports grants from NIH, during the conduct of the study. The authors report no other potential conflicts of interest for this work.

\section{References}

1. Friedman SM, Mulhausen P, Cleveland ML, et al. Healthy aging: American Geriatrics Society White Paper Executive Summary. $J$ Am Geriatr Soc. 2019;67(1):17-20. doi:10.1111/jgs.15644

2. Dzau VJ, Inouye SK, Rowe JW, Finkelman E, Yamada T. Enabling healthful aging for all - the National Academy of Medicine grand challenge in healthy longevity. $N$ Engl $J$ Med. 2019;381 (18):1699-1701. doi:10.1056/NEJMp1912298

3. Khaw KT. Healthy aging. BMJ. 1997;315(7115):1090-1096. doi:10.11 36/bmj.315.7115.1090

4. Haveman-Nies A, de Groot LC, van Staveren WA. Dietary quality, lifestyle factors and healthy ageing in Europe: the SENECA study. Age Ageing. 2003;32(4):427-434. doi:10.1093/ageing/32.4.427

5. Hamer M, Lavoie KL, Bacon SL. Taking up physical activity in later life and healthy ageing: the English longitudinal study of ageing. Br J Sports Med. 2014;48(3):239-243. doi:10.1136/bjsports-2013-092993

6. Sun Q, Townsend MK, Okereke OI, Franco OH, Hu FB, Grodstein F. Physical activity at midlife in relation to successful survival in women at age 70 years or older. Arch Intern Med. 2010;170(2):194-201. doi:10.1001/archinternmed.2009.503

7. Sun Q, Townsend MK, Okereke OI, et al. Alcohol consumption at midlife and successful ageing in women: a prospective cohort analysis in the nurses' health study. PLoS Med. 2011;8(9):e1001090. doi:10. 1371/journal.pmed.1001090 
8. Jackson AS, Janssen I, Sui X, Church TS, Blair SN. Longitudinal changes in body composition associated with healthy ageing: men, aged 20-96 years. Br J Nutr. 2012;107(7):1085-1091. doi:10.1017/ S0007114511003886

9. Ma W, Hagan KA, Heianza Y, Sun Q, Rimm EB, Qi L. Adult height, dietary patterns, and healthy aging. Am J Clin Nutr. 2017;106 (2):589-596. doi:10.3945/ajcn.116.147256

10. Kwok CS, Kontopantelis E, Kuligowski G, et al. Self-reported sleep duration and quality and cardiovascular disease and mortality: a dose-response meta-analysis. $J$ Am Heart Assoc. 2018;7(15): e008552. doi:10.1161/JAHA.118.008552

11. Itani O, Jike M, Watanabe N, Kaneita Y. Short sleep duration and health outcomes: a systematic review, meta-analysis, and meta-regression. Sleep Med. 2017;32:246-256. doi:10.1016/j.sleep. 2016.08.006

12. Jike M, Itani O, Watanabe N, Buysse DJ, Kaneita Y. Long sleep duration and health outcomes: a systematic review, meta-analysis and meta-regression. Sleep Med Rev. 2018;39:25-36. doi:10.1016/j. smrv.2017.06.011

13. Bubu OM, Brannick M, Mortimer J, et al. Sleep, cognitive impairment, and Alzheimer's disease: a systematic review and metaanalysis. Sleep. 2017;40(1). doi:10.1093/sleep/zsw032.

14. Devore EE, Grodstein F, Duffy JF, Stampfer MJ, Czeisler CA, Schernhammer ES. Sleep duration in midlife and later life in relation to cognition. J Am Geriatr Soc. 2014;62(6):1073-1081. doi:10.1111/jgs.12790

15. Zhai L, Zhang H, Zhang D. Sleep duration and depression among adults: a meta-analysis of prospective studies. Depress Anxiety. 2015;32(9):664-670. doi:10.1002/da.22386

16. Liu TZ, Xu C, Rota M, et al. Sleep duration and risk of all-cause mortality: a flexible, non-linear, meta-regression of 40 prospective cohort studies. Sleep Med Rev. 2017;32:28-36. doi:10.1016/j. smrv.2016.02.005

17. Shen X, Wu Y, Zhang D. Nighttime sleep duration, 24-hour sleep duration and risk of all-cause mortality among adults: a meta-analysis of prospective cohort studies. Sci Rep. 2016;6:21480. doi:10.1038/ srep21480

18. Yin J, Jin X, Shan Z, et al. Relationship of sleep duration with all-cause mortality and cardiovascular events: a systematic review and dose-response meta-analysis of prospective cohort studies. $J \mathrm{Am}$ Heart Assoc. 2017;6(9). doi:10.1161/JAHA.117.005947.

19. da Silva AA, de Mello RG, Schaan CW, Fuchs FD, Redline S, Fuchs SC. Sleep duration and mortality in the elderly: a systematic review with meta-analysis. BMJ Open. 2016;6(2):e008119. doi:10.1136/bmjopen-2015-008119

20. Buysse DJ. Sleep health: can we define it? Does it matter? Sleep. 2014;37(1):9-17. doi:10.5665/sleep.3298

21. Li M, Li K, Zhang XW, Hou WS, Tang ZY. Habitual snoring and risk of stroke: a meta-analysis of prospective studies. Int $J$ Cardiol. 2015;185:46-49. doi:10.1016/j.ijcard.2015.03.112

22. Li D, Liu D, Wang X, He D. Self-reported habitual snoring and risk of cardiovascular disease and all-cause mortality. Atherosclerosis. 2014;235(1):189-195. doi:10.1016/j.atherosclerosis.2014.04.031

23. Quesnot A, Alperovitch A. Snoring and risk of cognitive decline: a 4-year follow-up study in 1389 older individuals. J Am Geriatr Soc. 1999;47(9):1159-1160. doi:10.1111/j.1532-5415.1999.tb052 52.x

24. Knuiman M, James A, Divitini M, Bartholomew H. Longitudinal study of risk factors for habitual snoring in a general adult population: the Busselton Health Study. Chest. 2006;130(6):1779-1783. doi:10.1378/chest.130.6.1779

25. Sporndly-Nees S, Asenlof P, Theorell-Haglow J, Svensson M, Igelstrom H, Lindberg E. Leisure-time physical activity predicts complaints of snoring in women: a prospective cohort study over 10 years. Sleep Med. 2014;15(4):415-421. doi:10.1016/j. sleep.2013.09.020
26. Patel SR, Ayas NT, Malhotra MR, et al. A prospective study of sleep duration and mortality risk in women. Sleep. 2004;27(3):440-444. doi:10.1093/sleep/27.3.440

27. Stampfer MJ, Kang JH, Chen J, Cherry R, Grodstein F. Effects of moderate alcohol consumption on cognitive function in women. $N$ Engl J Med. 2005;352(3):245-253. doi:10.1056/NEJMoa041152

28. Brandt J, Spencer M, Folstein M. The telephone interview of cognitive status. Neuropsychol Behav Neurol. 1988;1:111-117.

29. Lee S, Kawachi I, Berkman LF, Grodstein F. Education, other socioeconomic indicators, and cognitive function. Am J Epidemiol. 2003;157(8):712-720. doi:10.1093/aje/kwg042

30. Ware JE, Sherbourne CD. The MOS 36-item short-form health survey (SF-36). I. Conceptual framework and item selection. Med Care. 1992;30(6):473-483. doi:10.1097/00005650-199206000-00002

31. Willett WC, Sampson L, Stampfer MJ, et al. Reproducibility and validity of a semiquantitative food frequency questionnaire. Am J Epidemiol. 1985;122(1):51-65. doi:10.1093/oxfordjournals.aje.a114086

32. Yuan C, Spiegelman D, Rimm EB, et al. Relative validity of nutrient intakes assessed by questionnaire, 24-hour recalls, and diet records as compared with urinary recovery and plasma concentration biomarkers: findings for women. Am J Epidemiol. 2018;187(5):1051-1063. doi:10.1093/aje/kwx328

33. Chiuve SE, Fung TT, Rimm EB, et al. Alternative dietary indices both strongly predict risk of chronic disease. J Nutr. 2012;142 (6):1009-1018. doi:10.3945/jn.111.157222

34. Sun Q, Townsend MK, Okereke OI, Franco OH, Hu FB, Grodstein F. Adiposity and weight change in mid-life in relation to healthy survival after age 70 in women: prospective cohort study. BMJ. 2009;339: b3796. doi:10.1136/bmj.b3796

35. Durrleman S, Simon R. Flexible regression models with cubic splines. Stat Med. 1989;8(5):551-561. doi:10.1002/sim.4780080504

36. Gildner TE, Liebert MA, Kowal P, Chatterji S, Snodgrass JJ. Associations between sleep duration, sleep quality, and cognitive test performance among older adults from six middle income countries: results from the Study on Global Ageing and Adult Health (SAGE). J Clin Sleep Med. 2014;10(6):613-621. doi:10.5664/jcsm.3782

37. Ayas NT, White DP, Al-Delaimy WK, et al. A prospective study of self-reported sleep duration and incident diabetes in women. Diabetes Care. 2003;26(2):380-384. doi:10.2337/diacare.26.2.380

38. Ayas NT, White DP, Manson JE, et al. A prospective study of sleep duration and coronary heart disease in women. Arch Intern Med. 2003;163(2):205-209. doi:10.1001/archinte.163.2.205

39. Qin Y, Zhou Y, Zhang X, Wei X, He J. Sleep duration and breast cancer risk: a meta-analysis of observational studies. Int J Cancer. 2014;134(5):1166-1173. doi:10.1002/ijc.28452

40. Xie L, Kang H, Xu Q, et al. Sleep drives metabolite clearance from the adult brain. Science. 2013;342(6156):373-377. doi:10.1126/ science. 1241224

41. Shokri-Kojori E, Wang GJ, Wiers CE, et al. beta-Amyloid accumulation in the human brain after one night of sleep deprivation. Proc Natl Acad Sci U S A. 2018;115(17):4483-4488. doi:10.1073/pnas.1721694115

42. Ooms S, Overeem S, Besse K, Rikkert MO, Verbeek M, Claassen JA. Effect of 1 night of total sleep deprivation on cerebrospinal fluid beta-amyloid 42 in healthy middle-aged men: a randomized clinical trial. JAMA Neurol. 2014;71(8):971-977. doi:10.1001/jamaneurol.2014.1173

43. Huang T, Redline S. Cross-sectional and prospective associations of actigraphy-assessed sleep regularity with metabolic abnormalities: the multi-ethnic study of atherosclerosis. Diabetes Care. 2019;42 (8):1422-1429. doi:10.2337/dc19-0596

44. Huang T, Mariani S, Redline S. Sleep irregularity and risk of cardiovascular events: the multi-ethnic study of atherosclerosis. $\mathrm{J} \mathrm{Am} \mathrm{Coll}$ Cardiol. 2020;75(9):991-999. doi:10.1016/j.jacc.2019.12.054

45. Moreno CRC, Marqueze EC, Sargent C, Wright KP, Ferguson SA, Tucker P. Working time society consensus statements: evidence-based effects of shift work on physical and mental health. Ind Health. 2019;57(2):139-157. doi:10.2486/indhealth.SW-1 
46. Vogel M, Braungardt T, Meyer W, Schneider W. The effects of shift work on physical and mental health. J Neural Transm. 2012;119 (10):1121-1132. doi:10.1007/s00702-012-0800-4

47. Vetter C, Devore EE, Wegrzyn LR, et al. Association between rotating night shift work and risk of coronary heart disease among women. JAMA. 2016;315(16):1726-1734. doi:10.1001/jama.2016.4454

48. Goto R, Tanigawa T, Maruyama K, et al. Associations of snoring frequency with blood pressure among the lean Japanese population: the Toon Health Study. J Hum Hypertens. 2020;34(4):271-277. doi:10.1038/s41371-0180148-9

49. Endeshaw Y, Rice TB, Schwartz AV, et al. Snoring, daytime sleepiness, and incident cardiovascular disease in the health, aging, and body composition study. Sleep. 2013;36(11):1737-1745. doi:10.5665/ sleep. 3140

50. Sands M, Loucks EB, Lu B, et al. Self-reported snoring and risk of cardiovascular disease among postmenopausal women (from the Women's Health Initiative). Am J Cardiol. 2013;111(4):540-546. doi:10.1016/j.amjcard.2012.10.039

51. Dalmasso F, Prota R. Snoring: analysis, measurement, clinical implications and applications. Eur Respir J. 1996;9(1):146-159. doi:10. 1183/09031936.96.09010146

52. Loke YK, Brown JW, Kwok CS, Niruban A, Myint PK. Association of obstructive sleep apnea with risk of serious cardiovascular events: a systematic review and meta-analysis. Circ Cardiovasc Qual Outcomes. 2012;5(5):720-728. doi:10.1161/CIRCOUTCOMES.11 1.964783

53. Raghuram A, Clay R, Kumbam A, Tereshchenko LG, Khan A. A systematic review of the association between obstructive sleep apnea and ventricular arrhythmias. J Clin Sleep Med. 2014;10 (10):1155-1160. doi:10.5664/jcsm.4126

54. Xu S, Wan Y, Xu M, et al. The association between obstructive sleep apnea and metabolic syndrome: a systematic review and meta-analysis. BMC Pulm Med. 2015;15:105. doi:10.1186/s12890015-0102-3

55. Qie R, Zhang D, Liu L, et al. Obstructive sleep apnea and risk of type 2 diabetes mellitus: a systematic review and dose-response meta-analysis of cohort studies. J Diabetes. 2020;12(6):455-464. doi:10.1111/1753-0407.13017

56. Palamaner Subash Shantha G, Kumar AA, Cheskin LJ, Pancholy SB. Association between sleep-disordered breathing, obstructive sleep apnea, and cancer incidence: a systematic review and meta-analysis. Sleep Med. 2015;16(10):1289-1294. doi:10.1016/j.sleep.2015.04.014
57. Gupta MA, Simpson FC. Obstructive sleep apnea and psychiatric disorders: a systematic review. J Clin Sleep Med. 2015;11 (2):165-175. doi:10.5664/jcsm.4466

58. Bubu OM, Andrade AG, Umasabor-Bubu OQ, et al. Obstructive sleep apnea, cognition and Alzheimer's disease: a systematic review integrating three decades of multidisciplinary research. Sleep Med Rev. 2020;50:101250.

59. Gelber RP, Redline S, Ross GW, et al. Associations of brain lesions at autopsy with polysomnography features before death. Neurology. 2015;84(3):296-303. doi:10.1212/WNL.0000000000001163

60. Goldstein AN, Walker MP. The role of sleep in emotional brain function. Annu Rev Clin Psychol. 2014;10:679-708. doi:10.1146/ annurev-clinpsy-032813-153716

61. Venkataraman S, Vungarala S, Covassin N, Somers VK. Sleep apnea, hypertension and the sympathetic nervous system in the adult population. J Clin Med. 2020;9(2):591. doi:10.3390/jcm9020591

62. Goya TT, Silva RF, Guerra RS, et al. Increased muscle sympathetic nerve activity and impaired executive performance capacity in obstructive sleep apnea. Sleep. 2016;39(1):25-33. doi:10.5665/ sleep. 5310

63. Flaa A, Aksnes TA, Kjeldsen SE, Eide I, Rostrup M. Increased sympathetic reactivity may predict insulin resistance: an 18-year follow-up study. Metabolism. 2008;57(10):1422-1427. doi:10.1016/ j.metabol.2008.05.012

64. Cespedes EM, Hu FB, Redline S, et al. Comparison of self-reported sleep duration with actigraphy: results from the hispanic community health study/study of latinos sueno ancillary study. Am J Epidemiol. 2016;183(6):561-573. doi:10.1093/aje/kwv251

65. Lauderdale DS, Knutson KL, Yan LL, Liu K, Rathouz PJ. Self-reported and measured sleep duration: how similar are they? Epidemiology. 2008;19(6):838-845. doi:10.1097/EDE.0b013e318187a7b0

66. Garfield V, Joshi R, Garcia-Hernandez J, Tillin T, Chaturvedi N. The relationship between sleep quality and all-cause, CVD and cancer mortality: the Southall and Brent REvisited study (SABRE). Sleep Med. 2019;60:230-235. doi:10.1016/j.sleep.2019.03.012

67. Baden MY, Hu FB, Vetter C, Schernhammer E, Redline S, Huang T. Sleep duration patterns in early to middle adulthood and subsequent risk of type 2 diabetes in women. Diabetes Care. 2020;43 (6):1219-1226. doi:10.2337/dc19-2371
Nature and Science of Sleep

\section{Publish your work in this journal}

Nature and Science of Sleep is an international, peer-reviewed, open access journal covering all aspects of sleep science and sleep medicine, including the neurophysiology and functions of sleep, the genetics of sleep, sleep and society, biological rhythms, dreaming, sleep disorders and therapy, and strategies to optimize healthy sleep.
The manuscript management system is completely online and includes a very quick and fair peer-review system, which is all easy to use. Visit http://www.dovepress.com/testimonials.php to read real quotes from published authors. 